\title{
Patterns-based Information Systems Organization
}

\author{
Sergej Lugović \\ Polytechnic of Zagreb \\ Vrbik 8, Zagreb, Croatia \\ slugovic@tvz.hr \\ Ivan Dunđer \\ Department of Information and Communication Sciences, \\ Faculty of Humanities and Social Sciences, University of Zagreb \\ Ivana Lučića 3, Zagreb, Croatia \\ idundjer@ffzg.hr \\ Marko Horvat \\ Polytechnic of Zagreb \\ Vrbik 8, Zagreb, Croatia \\ mhorvat1@tvz.hr
}

\section{Summary}

The socio-technical systems research paradigm is about the complexity of real situations. It confronts us with the quest for variables that could provide us with insight into the behavior of such systems. Their behavior emerges according to internal system properties and adaptation of the system to external conditions.

In our view, behavioral patterns are one of those particular variables since machines can recognize them and their dynamics. Based on the synthesis of three different theoretical frameworks, this paper proposes a concept of patterns-based information system organization. The authors built the concept on the Deacon discussion of theory of information, Hofkirchner's unified information theory and related system behavior, and Kelso's explanation of pattern creation processes in self-organizing systems. All three researchers have included patterns in their theoretical proposal.

According to this analysis of the existing theories and their synthesis, we conclude that in order to design machines that can automatically support new behavior, we have to analyze humans and machines as a complex whole with $d y$ namic relationships and emerging patterns as a dependent variable of behavior. By developing this theoretical concept, we establish a departure point for future research and search for different variables that correlate with pattern formation.

Keywords: information, patterns, self-organizing, information behavior, information systems 
A pattern is a message, and may be transmitted as a message. (Wiener, 1950) The transformation of noise back into signal is part of the game. (Cohen, 2006) Messages can be studied according to their form, content, goal, producers, and recipients. (Capurro, 2003)

\section{Introduction}

If patterns are messages and could transform as messages, first they have to be recognizable. In a recent massive scale experiment of Facebook users, that's exactly what happened (Kramer et al 2014). The pattern of user behavior changed according to the amount of positive or negative content in their feed. When users were exposed to more positive content, users posted more positive status updates. In this experiment, researchers manipulated the users' content and measured the amount of positive or negative words in their status updates. So by recognizing patterns of agent behavior and by intervening correlated variables, we can transmit patterns of behavior. It's interesting to look where user behavior comes from. Because the agent (FB user) didn't know about the intervention, it's fair to say this behavior is self-organized and emerged from the internal agent behavior and not from the surrounding environment.

According to (Camazine, 2003, p.7), in self-organizing biological systems, patterns emerge from internal interactions using only information stored locally, while in systems lacking self-organization, a supervisor, directives, or already existing patterns in the environment can impose order. Is this human(s) who changed emotional behavior self-organizing or lacking self-organization? In our view, it's the position of the observer observing phenomena. If we look it from outside the system, we can recognize reference to the environment and feedback, but if we look at it from inside (from the position of the user) not knowing about intervention, then we could recognize reference to internal interactions and information stored in the user cognitive system.

If we're analyzing dynamic patterns formation, there are two problems we have to deal with. One is how very large material components construct the pattern, and the other relates to how given patterns are constructed to accommodate different circumstances. The former could be "the problem of complexity of substance" and the latest "the problem of pattern complexity" (Kelso, 1997, p. 5). Or how Batson (1972, p. 20) writes, "[M]ental process, ideas, communication, organization, differentiation, pattern, and so on, are matters of form rather than substance." Pattern as a phenomenon to observe could provide us with a lens to analyze how substance is formed. And it's in the domain of information science since it's concerned with the form and organization of information and the underlying structures, a fundamental methodological stance Bates (1999) described as socio-technical.

There are two modes of apprehending our experience's structure, state description and process description. The former characterizes the world as sensed and later characterizes the word as acted upon. "The organism must develop correlations between goals in the sensed world and actions in the world of process" 
(Simon, 1962). The former provides criteria for identifying objects or process of relating dynamic pattern formations with circumstances, while it later provides the means for producing objects or describes how a dynamic pattern of behavior is constructed.

In the Facebook research, we could see limitations of analyzing social agents and technology agents separately. Do we observe the social agent or tools he uses? The research on socio-technical systems is dealing with the complexity of real situations rather than analyzing separated aspects (Ropohl, 1999). When a system is complex and dynamic, to understand the system, it's not enough to observe its parts because knowing properties of each doesn't give complete information about the system. Therefore, we must treat such a system as a whole. To understand the principles of such a system, feedback, or interaction of the parts isn't enough. What is important is the assumption that such a dynamic system has complex behaviors and that those behaviors can be goal-seeking forming dynamic patterns (Ashby, 1956, p. 55).

In our view, by analyzing patterns, which relate to complex behavior and dynamic interactions in a system composed of social and technical agents, we can get insight into how they emerge according to the reflection of the environment and internal properties of such a system. So, patterns become dependent variables of the socio-technical system behavior, including internal properties and environment states. By understanding how behavior is constructed according to the environment and internal norms stored in a system, we can comprehend the complexity of real situations.

This aligns with the socio-technical practice in information science, which calls for research efforts to analyze how communities use tools to access documents and create knowledge (Tuominen et al., 2005). We believe that in this approach we overcome the divide underlined in two cybernetics approaches, one of the first order and another of the second order. According to Geyer (1995), there are four main differences. The first one is dealing with the observer and the system where in the second-order cybernetics relationship between the observer and the system should be included in the analysis. The second one is that the second-order cybernetics is about constantly changing properties of the system, not its stability. In other words, it's about self-organization that isn't about forecasting but about understanding. The third is about the second-order cybernetics recognizing that all living organisms are self-steering and that their behavior can be steered from outside up to certain limits. The fourth is recognizing the continuous emergence of complexity resulting in new behavior and new interaction with the environment.

By observing patterns, we can take a step back from the system and observer relationship, and we can observe patterns in stable processes and in the changing ones. Different patterns will depend on steering from the outside and/or on internal system capabilities. By applying independent and objective machine observation, we can precisely observe the dynamics of those pattern emer- 
gences. What's important, in our view, is that patterns can be easily described by machine quantitatively, making them different from behavior, which is qualitative and hard to recognize by machines.

For the purpose of further discussion and in regard to conceptual development, we define patterns as a time sequence happening between the moment when an agent experiences information (i.e., when structured data become information) and the information-searching and seeking process starts until the moment when the agent stops interaction with the structured data as his information needs are satisfied. Such a process can occur in social and technical domains. This is a working definition describing the scope of the analysis.

\section{Self-organization and socio-technical systems}

Can we call socio-technical systems self-organizing? Humans live in the technotope, surrounded by technologies and material context, including technical artifacts, which are not only neutral, but they shape our perceptions, behavioral patterns, and activities (Geels, 2004). As the information society develops in terms of blurring the difference between online and offline, we're becoming inforgs ${ }^{1}$, socially connected information organisms, which will compete with other artificial inforgs. And this isn't happening because of the transformation of our bodies, but through re-ontologization (a radical form of reengineering) of our environment (Floridi, 2009).

So, to respond to changes in the environment we have to design and use technologies to adapt better, and by doing so, we have to move from our point of observing social or technical entities separately to observing socio-technical systems. Socio-technical systems are complex and behave in the new environment by developing new properties based on internal information processing capabilities. Such systems are self-organized since they don't have an internal agent doing the organization, but they form dynamic patterns of its behavior (Kelso 1997, p. 8). There's no agent defining the exact pattern of how author uses this machine while writing this text, but the usage pattern emerges according to cognitive, affective, social, and technical variables relationships.

We can discuss four specific properties of complex systems in terms of sociotechnical systems. The first is non-determinism since it's impossible to fully understand the system's behavior by knowing only its constituting functions. The second is limited functional decomposability because it's a dynamic system, and it isn't possible to study the system's properties by decomposing it into functional parts. Its interaction with the environment and the self-organizing property allow it to functionally restructure itself. The third is the distributed nature of information and representation; some of the system functions cannot

\footnotetext{
${ }^{1}$ Inforgs can be part of a hybrid agent that is, for example, a family with digital devices such as digital cameras, cell phones, tablets, and laptops. (Floridi, L. Ed. (2010). The Cambridge handbook of information and computer ethics. Cambridge University Press).
} 
be precisely localized. This is the cause of socio-technical system properties comparable to distributed systems. The fourth is the self-organization related to the emergent properties that we can't comprehend by understanding the components (Pavard and Dugdale, 2006).

We cannot know how a human and a mobile phone will behave by knowing only the phone's functions. Also, we can't understand what functions a user can execute on a mobile phone by knowing only the phone's functions. By adding more users with their mobile phones, a bigger system emerges, which is more difficult to understand regarding its structure, functions, behavior, and goals. Recent examples of self-organization of socio-technical systems based on users and mobile phones include the Arab Spring movements or in the rising popularity of mobile banking in Africa.

Another example of self-organizing socio-technical systems is the rise of the popularity of Bitcoin. Such a self-organization has many technical capabilities to process data, but a socio-cognitive and affective aspect will give meaning to that data and affect how patterns will form. Also, such a self-organization must expand in terms of selection and understanding how such "self-ordered properties permit, enable and limit the efficacy of natural selection" (Kauffman, 1993, p. xiv). By understanding selection along with self-organization, we have better insight into the process of new function emergence and what the correlations with self-organizing properties are.

Also, it's important to make a distinction between determinism and causality. When systems are far from an equilibrium and far from a deterministic clockwork state, they start to show self-organizing capabilities by following three propositions. The first one is there is no transformation mechanism that unambiguously turns cause to effect. Cause and effect relations allow a different cause to have the same effect and the same cause to have a different effect. The second one is that little changes in the cause may lead to a big change in effect. The third is that the more complex a system is the less probable is the return of the certain state in the future (Hofkirchner, 1998). Or to cite Hofkirchner (2011), "self-organization may be looked upon as the way evolutionary systems come into existence or change their structure, state or behavior and the way they maintain themselves (their structure, state or behavior)."

As stated by Kelso (1997, p. 32), patterns arise spontaneously as the result of interacting components, and the nature of such interaction is nonlinear and in such systems the whole is more and/or different from the sum of its parts. We can trace these ideas back to Immanuel Kant who pointed out that on one hand, organisms will always resist complete mechanistic and mathematical explanations since they have certain goal directedness (purpose), which humans can describe only in functional terms. On the other hand, biology as a science requires a mechanistic approach using forward causal explanation that needs to be expressed in mathematical terms. Kant stressed that the necessity of this dual ap- 
proach for studying organisms is not a property of nature but rather our limited faculties (Roth, 2011).

If we're about to design machines to help us process or compute information, this is a very important point. If we're trying to describe machines by these functional limitations, then machines by themselves will have functional disabilities. And if such machines are essential parts of the self-organizing sociotechnical systems, then, this dysfunction is a dysfunction of the socio-technical systems. If we could better describe the reality of nature, we could design machines that are more intelligent, and such machines would be part of the sociotechnical systems making them more intelligent too.

In our view, the social "half" of such a system is extremely important because the self-steering capacity of humans provides essential non-deterministic properties of such a system built on social and technical components. So, to have a more intelligent system, we have to overcome the problem of dualism in explaining nature.

Can we overcome this problem of dualism in explaining nature? We think that by observing dynamics of patterns as dependent variables of socio-technical systems, which emerge in self-organizing processes of such systems, we could open up new perspectives in different disciplines in information and computer science. The emphasis is on the dynamic patterns emergence, not static patterns. As they emerge, they aren't reducible to a substance from which they emerge because they have their own properties. These properties relate to the purpose of the higher order level, while the mechanics of their formations are at the level below (Wilson, 2002). And they emerge as a result of the process of organizing the observed system's internal properties and as a reflection of the environment's circumstances.

So, patterns as phenomena observed in the behavior of self-organizing sociotechnical systems can lead us to know about how these systems behave, including mechanical principles and principles related to the purpose of such systems. Patterns, as phenomena that correlate a mechanical view and a purpose view, can present behavior that's, we believe, qualitative and can create a mechanical structure that's quantitative. With development of computational technologies, we can observe such patterns emergence processes with extensive support of the machines, obtaining objective insights about how they form and what their dynamic is, according to internal or external factors influencing their formation.

So, we could use machines to help us observe dynamic pattern emergence, use them as a dependent variable in the research of socio-technical systems. By using those findings, we could design better machines conditioned by social selfsteering forces based on information needs. Such a force is implemented through the feedback, creating dynamic adaptation of those machines in a dialectic process (thesis, antithesis, and synthesis). 
In addition to the above-mentioned authors, we can find further socio-technical systems concept elaboration in the work of one of the founders of this paradigm Trist (1981) and a PhD thesis written by Shields (2007) analyzing different theories and practices used to study technology systems. An important contribution to the socio-technical system paradigm are the books The Social Construction of Technological Systems: New Directions in the Sociology and History of Technology (Bijker et al., 1981) and Science in Action: How to Follow Scientists and Engineers Through Society (Latour, 1987). Current research approaches the socio-technical system from the evolutionary perspective and is in the work of Heylighen (2007).

\section{Information, Systems, Organization, and Patterns}

In this part of the paper, we select and propose existing theoretical frameworks that support our views on patterns-based information system organization. We combined three existing theoretical frameworks related to socio-technical systems and information, offering theoretical fundamentals for proposal of a new synthesis, one that will support the design of machines that could automatically support new behavior of the socio-technical systems.

\section{Information and patterns}

Deacon (2010) discussed the information from the semiotics perspective, pointing out that information could refer to the sign or signal functioning without any meaning or reference, such as information content in bits. Such information is syntactic information. Information could also represent meaning of the bits from syntactic information. Such information could be semantic information. Information also could refer to the aspects of those ideas, which we call pragmatic information (Deacon, 2010). Syntactic information's basis is the Shannon theory of information; the semantic is from the Boltzmann and Shannon entropy, while the pragmatic one expands it with the theory of evolution. In the same work, Deacon pointed out that "both the patterns that we deliberately create in order to convey an idea and those we discover in nature can convey information. Ultimately, this demonstrates that almost anything can qualify as information in the syntactic sense, because this is only an assessment of the potential to inform." And such a basic notion of information corresponds to the Shannon theory of information. So, a pattern could be looked as a sign, which when recognized, could provide a basic component of the message transferred through the communication channel from the receiver to the sender. Here, we see that a pattern could be looked as a fundamental property of the information. To remove uncertainty we have to recognize the pattern and send it as a message to the receiver. Those three types of information are in Table 1 (adapted from Dodig-Crnkovic, 2012). 
Table 1. Deacon's three types of information

\begin{tabular}{|c|c|c|c|}
\hline $\begin{array}{c}\text { Type of infor- } \\
\text { mation }\end{array}$ & Underlying theories & Focus on & $\begin{array}{c}\text { Semiotics } \\
\text { perspectives }\end{array}$ \\
\hline Information 1 & Shannon & $\begin{array}{c}\text { Data, pattern, signal, data } \\
\text { communications }\end{array}$ & $\begin{array}{c}\text { Syntax } \\
\text { (what it exhibits) }\end{array}$ \\
\hline Information 2 & Shannon + Boltzmann & $\begin{array}{c}\text { Intentionality, "aboutness," } \\
\text { reference, representation, } \\
\text { relation to object or referent }\end{array}$ & $\begin{array}{c}\text { Semantics } \\
\text { (what it conveys) }\end{array}$ \\
\hline Information 3 & $\begin{array}{c}\text { Shannon + Boltzmann } \\
\text { + Evolution }\end{array}$ & $\begin{array}{c}\text { Function, interpretation, use, } \\
\text { pragmatic consequence }\end{array}$ & $\begin{array}{c}\text { Pragmatics } \\
\text { (what it is for) }\end{array}$ \\
\hline
\end{tabular}

\section{Systems and patterns}

Hofkirchner (1999) used semiotics as a basis for discussion about systems and information behavior, pointing to the concept of Peirce's triad of syntactic, semantic, and paradigmatic sign aspects. The information process consists of drawing distinctions in terms of system organizing based on those distinctions, whether it's in the new system that emerges or the novelty in the existing system.

Table 2. Three different types of system formation (based on Hofkirchner, 1999)

\begin{tabular}{|l|l|l|}
\hline \multicolumn{1}{|c|}{ Type of system } & Evolution of sign processes & \multicolumn{1}{|c|}{$\begin{array}{c}\text { System formations: } \\
\text { from patterns to new goals }\end{array}$} \\
\hline $\begin{array}{l}\text { Self-restructuring } \\
\text { systems }\end{array}$ & $\begin{array}{l}\text { Reflective pattern formation } \\
\text { in dissipative systems - } \\
\text { emergence of signs }\end{array}$ & $\begin{array}{l}\text { Forming patterns is the way self-restructuring } \\
\text { systems reflect some changes in the conditions } \\
\text { in the system's environment. }\end{array}$ \\
\hline $\begin{array}{l}\text { Self-reproducing } \\
\text { systems }\end{array}$ & $\begin{array}{l}\text { Intelligent symbolization in } \\
\text { autopoietic dissipative } \\
\text { systems - emergence of } \\
\text { symbols }\end{array}$ & $\begin{array}{l}\text { Self-reproduction requires structures to } \\
\text { functionalize for survival. Functionalized } \\
\text { structures are not plain patterns any more but } \\
\text { something that contains meaning. }\end{array}$ \\
\hline $\begin{array}{l}\text { Self-determining } \\
\text { systems }\end{array}$ & $\begin{array}{l}\text { Conscious formation of } \\
\text { ideas in recreative } \\
\text { autopoietic systems - } \\
\text { emergence of goals (ideas) }\end{array}$ & $\begin{array}{l}\text { Systems that do not merely (re)produce } \\
\text { themselves and strive for survival, but in doing } \\
\text { so, seek additional goals, which they're } \\
\text { committed to and which they chose on their } \\
\text { own. There are three steps of idea formation: } 1 . \\
\text { Perception of signals from outside the system } \\
\text { resulting in modification of the system } \\
\text { structure; 2. Interpretation of the perceptions by } \\
\text { which a system is modified; 3. Evaluation of the } \\
\text { interpretations modifying a system by affecting } \\
\text { behavior. }\end{array}$ \\
\hline
\end{tabular}

In realizing those novelties, a distinction is drawn and information produced. So, there exists a relationship between the old and the new sign; the new sign isn't reducible to the old sign but is dependent on it. Since we have a relationship of signs, we can refer to this as syntactic relations of signs. As a new state of the system also relates to the system's environment, a new relationship develops between the sign and the environment, and the sign gains significance, 
forming a semantic relation of signs (having meaning). As the sign gains significance, it can also relate to the aims of the system. We can call such a relationship a pragmatic relationship of signs (Hofkirchner, 1999). Such an analysis of different sign relations in self-organizing systems could give insight into how systems behave.

In Table 2, we present a brief summary of different system properties, the evolution of sign processes, and how a system forms itself in the process of self-organization.

\section{Organization and patterns}

Kelso proposes The Tripartite Scheme with the aim to theoretically summarize laws constituting the order parameter dynamics. The idea proposed is straightforward, proposing that understanding any level of organization starts with knowledge about three main things: parameters acting on the system, interacting elements of the system, and emerging patterns. There's also a clear point that this model is not rigid in terms of micro and macro (Kelso, 1997, p. 18). We present the Tripartite Scheme in Table 3.

Table 3. The Tripartite Scheme (based on Kelso, 1997)

\begin{tabular}{|l|l|}
\hline \multicolumn{1}{|c|}{ Knowledge about } & \multicolumn{1}{c|}{ Description } \\
\hline Cooperativities & The emerging patterns or modes to which they give rise \\
\hline Set of primitives & The interacting elements themselves \\
\hline Boundary conditions & The parameters acting on the system \\
\hline
\end{tabular}

\section{Synthesis}

We could clearly see that three theoretical models presented above have patterns in common. Deacon's model is dealing with information, the Hofkirchner's with systems, and the Kelso's with organization. We could synthesize those theoretical proposals into the concept of patterns-based information systems organization. Such a concept could be presented through three layers of self-organisaion:

- Structure level - the most simple biological self-organizing system (patterns formation according to internal properties) or simple self-restructuring mechanical systems (such as a PC that self-restructures data on a hard disk) making it applicable to socio-technical system analysis. Such a system is self-restructuring when the patterns emergence forms new structures, information explained in terms of the Shannon information theory and it organized around emerging dynamic patterns, the essence of cooperation.

- Function level - the autopoietic biological self-organizing system, which can reproduce itself, and to the intelligent mechanical system, which can reproduce its components to maintain a predefined goals execution process, making it applicable for socio-technical system analysis. Such a 
system is self-reproducing in terms of functional structures; information is defined in terms of Shannon and Boltzmann entropy, which explains intentionality, "aboutness," and reference to the information in the communication channel. And it organizes around interactions of elements for which prerequisite are formations of cooperatives.

- Goal level - the conscious socio-technical system, which can intervene in the environment and create new goals, according to its ideas. Such a system is self-determining, when perception determines new goals, interpretation of those perceptions, and evaluation of the interpretations results in the new behavior. Information is defined in terms of evolutionary principles describing use, functions, and pragmatic consequences. And it's organized according to the boundary conditions producing parameters acting on the system.

We can see that the last application of patterns-based information systems organization cannot separate into biological and mechanical systems. This leads us to the proposition that if we want to have machines to support new behavior of social structures by developing new structures, functions and new goals, we cannot analyze machines and humans separately, but as a complex whole with dynamic relationships and emerging patterns as a dependent variable of behavior.

Another condition to achieve such properties of the system is that the system should not have one central agent to control this process because it's selfregulated. It's also important to underline ethical implications related to such a process as an important dimension in further research.

If we want to gain the insight into how the system behavior emerges and how such a system performs in reshaping the environment according to its goals, what is observable are the dynamic patterns, with an emphasis on the dynamic. Therefore, if we take a point of observation from outside the system, we can see its performance, but we cannot gain insight into the emergence of particular behavior, which caused such a performance. By observing the system from the inside, we can understand the system properties and the emergence of behavior, but we can't see the result of such behavior.

Stepping back and observing the process of patterns formation as a dependent variable of system behavior and the system environment, we can learn how they emerge and how they relate to system performance. And such a position of observation is objective and considers the internal system behavior and reflection to the system environment.

Hjørland pointed out the 'tendency to try to measure the users' information need by questioning them or by studying their behavior, seems to be mistaken. What information is needed to solve a given problem is not primarily a psychological question, but a theoretical/philosophical one" (Hjørland, 2002). 
In our view, the concept of patterns-based information systems organization, is overcoming such pitfalls since patterns are more objective and quantitative then psychological, theoretical, or philosophical questions.

\section{Conclusion}

In this paper, we provided an overview and analysis of some of the existing theories about self-organizing systems, how they interpret information and how they organize it. It's important to state that in this work we did not focus exclusively on the fields of patterns recognition and analysis from the perspective of computer science. Our analysis aims at searching for the explanations of dynamic patterns formation in different types of self-organizing, socio-technical systems from the perspective of information science.

Consequently, we synthesized theoretical frameworks into the concept of patterns-based information systems organization. We select those theoretical frameworks in terms that all three of them address patterns. We did not base selection on systematic literature reviews but with the aim to support concept construction. Such a conceptualization provides the point of departure toward future research on additional variables, which correlate with the patterns as behavior-dependent variables of socio-technical systems.

As a part of future research, our next task is to investigate existing empirical studies concerning patterns in socio-technical systems, including theoretical foundations. We believe that an aggregate investigation of the information behavior, information systems with pattern recognition, and application of cybernetics principles of feedback and self-organization could lead to new, encouraging and interdisciplinary research perspectives.

\section{References}

Ashby, W. R. (1956). An introduction to cybernetics (Vol. 2). London: Chapman \& Hall.

Bates, M. J. (1999). The invisible substrate of information science. Journal of the American Society for Information Science, 50(12), (1999). 1043-1050.

Bateson, G. (1972). Steps to an ecology of mind: Collected essays in anthropology, psychiatry, evolution, and epistemology. University of Chicago Press.

Bijker, W. E., Hughes, T. P., Pinch, T., \& Douglas, D. G. (2012). The social construction of technological systems: New directions in the sociology and history of technology. MIT press.

Camazine, S. (2003). Self-organization in biological systems. Princeton University Press.

Capurro, R. (2003). Angeletics - A Message Theory. Hierarchies of Communication. Karlsruhe: ZKM-Center for Art and Media, 58-71.

Cohen, I. R. (2006). Informational landscapes in art, science, and evolution. Bulletin of Mathematical Biology, 68(5), 1213-1229.

Deacon, T. W. (2010). What is missing from theories of information? Information and the Nature of Reality, 146, chapter 8.

Dodig-Crnkovic, G. (2012). Physical computation as dynamics of form that glues everything together. Information, 3(2), 204-218, citing Deacon, T. W. (2011). Incomplete nature: How mind emerged from matter. WW Norton \& Co. 
Geels, F. W. (2004). From sectoral systems of innovation to socio-technical systems: Insights about dynamics and change from sociology and institutional theory. Research policy, 33(6), 897-920.

Geyer, F. (1995). The challenge of sociocybernetics. Kybernetes: The International Journal of Systems \& Cybernetics, 24(4), 6-32.

Floridi, L. (2009). Web 2.0 vs. the semantic web: A philosophical assessment. Episteme, 6(01), 25-37.

Heylighen F. (2007): Accelerating Socio-Technological Evolution: from ephemeralization and stigmergy to the global brain, Globalization as an evolutionary process: modeling global change, eds. Modelski, G., D. Tessaleno, \& Thompson, W. London: Routledge.

Hjørland, B. (2002). Domain analysis in information science: eleven approaches-traditional as well as innovative. Journal of documentation, 58(4), 422-462.

Hofkirchner, W. (1998). Emergence and the logic of explanation. An argument for the unity of science. Acta Polytechnica Scandinavica, Mathematics, Computing, and Management in Engineering Series, 91, 23-30.

Hofkirchner, W. (1999). Towards a unified theory of information - The merging of second-order cybernetics and semiotics into a single and comprehensive information science. In 15e Congrès International de Cybernétique, Namur 1998, Namur 1999, 175-180.

Hofkirchner, W. (2011). Does computing embrace self-organisation. Information and computation: Essays on scientific and philosophical understanding of foundations of information and computation. Vol. 2. (edited by Gordana Dodig-Crnkovic and Mark Burgin).

Kauffman, S. A. (1993). The origins of order: Self-organization and selection in evolution. Oxford University Press.

Kelso, J. S. (1997). Dynamic patterns: The self-organization of brain and behavior. MIT press, Kramer, A. D., Guillory, J. E., \& Hancock, J. T. (2014). Experimental evidence of massivescale emotional contagion through social networks. Proceedings of the National Academy of Sciences

Latour, B. (1987). Science in action: How to follow scientists and engineers through society. Harvard University Press.

Pavard, B. and Dugdale, J. (2006). The contribution of complexity theory to the study of sociotechnical cooperative systems. Unifying Themes in Complex Systems. Springer Berlin, Heidelberg.

Ropohl, G. (1999). Philosophy of socio-technical systems. Techné: Research in Philosophy and Technology, 4(3), 186-194.

Roth, S. (2011). Mathematics and biology: a Kantian view on the history of pattern formation theory. Development genes and evolution, 221(5-6), 255-279.

Simon, H. A. (1962). The architecture of complexity. Proceedings of the American Philosophical Society, 106(6), 467-482.

Shields, W. M. (2007). Theory and practice in the study of technological systems. Doctoral dissertation, Retrieved from Virginia Polytechnic Institute and State University.

Trist, E. (1981). The evolution of socio-technical systems. Ontario Quality of Work Life Centre, (2), Ontario, Canada.

Tuominen, K., Savolainen, R. and Talja, S. (2005). Information literacy as a sociotechnical practice, The Library Quarterly 75(3), p329-345.

Wiener, N. (1988). The Human Use of Human Beings: Cybernetics and Society (No. 320). Da Capo Press.

Wilson, T. (2002). "Information science" and research methods. Knižnicná a informacná veda [Library and Information Science], XIX, 63-71. (Published by the Department of Library and Information Science, Comenius University, Bratislava, Slovak Republic. 